Including information on the therapeutic window in bioequivalence acceptance Peer-reviewed author version

JACOBS, Tom; De Ridder, Filip; Rusch, Sarah; Van Peer, Achiel; MOLENBERGHS, Geert \& BIJNENS, Luc (2008) Including information on the therapeutic window in bioequivalence acceptance. In: PHARMACEUTICAL RESEARCH, 25(11). p. 2628-2638.

DOI: $10.1007 / \mathrm{s} 11095-008-9680-6$

Handle: http://hdl.handle.net/1942/8542 


\title{
Including information on the therapeutic window in bioequivalence acceptance limits
}

\author{
Tom Jacobs ${ }^{1}$, Filip De Ridder ${ }^{2}$, Sarah Rusch², Achiel Van Peer ${ }^{2}$, \\ Geert Molenberghs ${ }^{1}$, Luc Bijnens ${ }^{2}$ \\ ${ }^{1}$ Hasselt University, Center for Statistics, Agoralaan 1, B-3590 Diepenbeek, Belgium \\ ${ }^{2}$ Johnson and Johnson Pharmaceutical Research and Development, \\ a division of Janssen Pharmaceutica N.V. Turnhoutseweg 30, B-2340 Beerse, Belgium
}

\section{SUMMARY}

Pharmaceutical companies use pharmacokinetic measurements in bioequivalence (BE) trials as surrogate to prove that a new drug formulation or manufacturing procedure does not alter the safety and efficacy profile of the drug. In general, Health Authorities require that the $90 \%$ confidence intervals about the geometric mean test/reference ratios for both $C_{\max }$ and $A U C$ fall between $80-125 \%$ to accept bioequivalence. For highly variable drugs and drug products, a high number of subjects is required in clinical trials to meet the current BE standards. Boddy (1995) and Karalis (2004, 2005) published approaches to correct or to widen, respectively, the acceptance ranges accounting for the degree of within-subject variability.

In 2006, Health Canada released a guidance on bioequivalence requirements for critical dose drugs and proposed more stringent acceptance limits of $90-112 \%$ for $A U C$. In that guidance, critical dose drugs are defined as those where small differences in dose or concentration lead to serious therapeutic failures and/or serious adverse drug reactions.

In this work, the approach of Karalis is extended to adapt the BE acceptance ranges to the therapeutic window of the drug, quantified as the ratio of the Maximum Tolerated Dose/Therapeutic Dose (MTD/D) and the Therapeutic Dose/Least Effective Dose (D/LED). A series of simulations was carried out to assess the performance of the adapted acceptance range in a two-treatment, two-period cross-over study, with different sample sizes $(12,24$ or 36 subjects), within-subject variabilities (15, 35 and $55 \% \mathrm{CV}$ ) and various ratios of MTD/D and D/LED. In addition, the method was retrospectively applied to the phenytoin data of Meyer (2001), theophylline data of Mistry (1999), and digoxin data of Martin (1997).

The results show that the approach has the desirable property of resulting in a more narrow acceptance range for doses near the boundaries of the therapeutic window and a wider acceptance range for products with a broad therapeutic window. 
Keywords: average bioequivalence; bioavailability; individual bioequivalence; therapeutic window.

\section{Introduction}

Bioequivalence studies are important in drug development to prove that two drug products give similar exposure in a subject, and therefore that the safety and efficacy profile is not altered and therapeutic equivalence can be claimed. Bioavailability and bioequivalence studies are performed to evaluate differences in drug products, for example research versus market tablets, various batches, or production sites. At the same time, those techniques are also used for evaluating food effects, drug-drug interactions, and comparing administration routes.

Schuirmann (1987) laid the foundations of modern bioequivalence testing. He proposed to perform two one-sided tests, to test the hypothesis that the ratio of the key pharmacokinetic parameters $A U C$ and $C_{\max }$ is contained within a prespecified range, which usually is $80-125 \%$. At the end of the twentieth century, average bioequivalence as proposed by Schuirmann was questioned because it only focusses on whether the average exposure of the study population is equivalent (Anderson and Hauck, 1990, Scheiner 1992). In the typical situation where drugs are on the market, each patient should maintain the same exposure independent of his choice. This led to the concept of individual bioequivalence (Anderson and Hauck, 1990), also known as switchability. Owing to the complexity of the technique and its favoring of highly variable drug products (Hsuan 2000), individual bioequivalence has not been used extensively to date.

There are two situations in which the traditional approach with a fixed acceptance range is not optimal: first the one of highly variable drug products, and secondly narrow index drugs, i.e., drugs where comparatively small differences in dose or concentration lead to dose-and concentration-dependent, serious therapeutic failures and/or serious adverse drug 
reactions.

An area of discussion is the bioequivalence assessment of highly variable drug products, i.e., products with a within-subject variability of more than $30 \%$. Authorities acknowledge that the large sample sizes for trials with such drug products cannot always be ethically justified (FDA 2003, CPMP EMEA 2006). The simplest correction for highly variable drug products, is by extending the acceptance limits from 80-125\% to $75-133 \%$ (CPMP EMEA 2001). Boddy (1995) proposed to modify the limits for highly variable drug products according to a predefined estimate of the within-subject variability of the reference drug product. The disadvantage of the $30 \%$ threshold is a discontinuity at that threshold: For example, it is possible that in a given study, a within-subject variability of $29 \%$ is observed and no modification of the limits is applied, while if the variability was slightly more than $30 \%$, adaptation of the acceptance limits could have yielded to a different conclusion.

Karalis et al. (2004, 2005) modified the idea of extending the bioequivalence limits. Whereas Boddy et al. (1995) categorize drug substances according to a within-subject variability of less versus more than $30 \%$, Karalis expands the bioequivalence limits in a continuous fashion as a function of the within-subject variability. However, expanding the acceptance limits increases the risk of false positives, i.e., falsely concluding two drug products to be bioequivalent. Therefore, Karalis proposed to incorporate the observed geometric mean ratio of the pharmacokinetic parameters $A U C$ and $C_{\max }$ in the acceptance limits: the further the geometric mean ratio deviates from equality, the more conservative the acceptance range becomes.

As suggested by the FDA guidance, the therapeutic window should be taken into account instead of performing an automatic extension of the acceptance ranges:

"Where the test product generates plasma concentrations that are substantially above those of the reference product, the regulatory concern is not therapeutic failure, but the adequacy of the safety database from the test product. Where the test product has plasma concentrations that are substantially below those of the 
reference product, the regulatory concern becomes therapeutic efficacy. When the variability of the test product rises, the regulatory concern relates to both safety and efficacy, because it may suggest that the test product does not perform as well as the reference product, and the test product may be too variable to be clinically useful." (FDA 2003)

The aim of the research in this paper is to present further approaches in bioequivalence acceptance taking into account the therapeutic window as suggested by the guidelines (FDA 2003). The proposed bioequivalence limits in this paper consider the position of the therapeutic dose with respect to the least effective dose $(L E D)$ and the maximum tolerated dose $(M T D)$. A dose close to the $L E D$ and/or the $M T D$ may require more stringent limits ensuring exposure remains within the therapeutic window.

The paper is organized as follows. First, the methodology for expanding the bioequivalence limits is described in Section 2. The performance of the method as evaluated through simulations is described in Section 3. Finally the method is applied to three known examples of narrow index drugs (theophylline, digoxin, and phenytoin) in Section 4.

\section{Methodology}

Let us first introduce some notation: $U$ and $L$ are the upper and lower acceptance limit, $\alpha$ the traditional limit $(125 \%), \beta$ the extended limit $(143 \%), \Psi$ the geometric mean ratio, $\sigma_{w}$ the within-subject standard deviation, $\gamma, \delta$, and $\theta$ are rate constants. $D$ is the therapeutic dose, which usually corresponds more or less to the administered dose, however, the phenytoin example is an example where the administered dose is lower than the therapeutic dose.

A first approach to adapt the bioequivalence limits for studies with highly variable drug substances was introduced by Boddy (1995). His method maintains the original method and acceptance ranges proposed by Schuirmann (1987) for drug substances with a low variability, i.e., $\% C V<30 \%$. For drug substances with a higher variability, the acceptance ranges are rescaled using the within-subject variability, with the $90 \%$ confidence interval of 
the difference on the logarithmic scale satisfying the criterion:

$$
\left|\mu_{T}-\mu_{R}\right| \leq \vartheta \sigma_{w}
$$

where the left side of the expression is the treatment difference on the logarithmic scale, $\sigma_{w}$ the within-subject standard deviation, and usually $\vartheta=1$. This means there is a discontinuation in the acceptance ranges at a within-subject variability of $30 \%$.

Karalis et. al. (2005) tried to overcome the discontinuity and proposed three types of bioequivalence limits depending on the geometric mean ratio and at the same time rescaling according to the within-subject variability in a continuous manner. In this paper Weibull type limits will be used to further refine the proposed approach of Karalis:

$$
U=\alpha+(5-4 \Psi)(\beta-\alpha)\left\{1-e^{-\left(\gamma \sigma_{w}\right)^{2}}\right\}
$$

with $\gamma$ a constant to regulate the expansion of the acceptance limit, the lower acceptance limit $L$ is $1 / U$. For low within-subject variability and $\Psi=1$, the upper limit remains approximatively $\alpha$, whereas for large variability and $\Psi=1$, the upper limit approximates $\beta$. With $\Psi=\alpha$, the upper limit is fixed to $\alpha$, regardless of the variability. Its sigmoid behavior ensures that the acceptance ranges remain almost unaffected under small variability, in contrast to the exponential and Michaelis-Menten type corrections (Karalis 2005). The continuity of the technique makes it also more appealing than the proposal of Boddy (1995).

A more general formulation is

$$
U=\alpha+5\left(1-\frac{1}{\alpha} \Psi\right)(\beta-\alpha)\left\{1-e^{-\left(\gamma \sigma_{w}\right)^{2}}\right\} 1_{\Psi \leq \alpha},
$$

with $L=1 / U$ as before. Using $\alpha=125 \%$ in the above equation simplifies to $(2.2) .1_{\Psi \leq \alpha}$ is added to indicate explicitly that $\Psi$ should fall within the acceptance range. As the focus is not on the choice of $\gamma$, it will be fixed in the rest of the paper to a value of 3 . This restricts by no means the results of the paper and is mainly chosen based on the simulations from Karalis (2005) to ensure that the acceptance ranges remain close to the standard 80-125\% for small variabilities.

In this paper, the expansion of the acceptance range will not only depend on the within-subject variability, but will also depend on the therapeutic window. Therefore, a 
second correction factor, which represents a similar sigmoidal function of the therapeutic window is added.

$$
\begin{aligned}
U & =\alpha+5\left(1-\frac{1}{\alpha} \Psi\right)(\beta-\alpha)\left\{1-e^{-\left(\gamma \sigma_{w}\right)^{2}}\right\}\left\{1-e^{-\left(\delta \frac{M T D}{D}\right)^{2}}\right\} 1_{\Psi \leq \alpha}, \\
L & =\frac{1}{\alpha+5\left(1-\frac{1}{\alpha} \Psi\right)(\beta-\alpha)\left\{1-e^{-\left(\gamma \sigma_{w}\right)^{2}}\right\}\left\{1-e^{-\left(\delta \frac{D}{L E D}\right)^{2}}\right\} 1_{\Psi \geq \alpha}} .
\end{aligned}
$$

The therapeutic window is defined as the ratio $D / L E D$ and the ratio $M T D / D$. Note the asymmetric character of the acceptance limits: the lower limit depends on the distance between the dose and the $L E D$, whereas the upper limit depends on the distance between the dose and the $M T D$.

A more conservative approach can be applied for narrow-index drugs. The concern has been introduced in the Canadian guideline (Ministry of Health Canada, 2006) that for certain drugs the 80-125\% acceptance range would be too liberal. Therefore, the standard $125 \%$ limit, which is used as a starting point in the current approach, can be modified in a similar way. This renders the resulting acceptance ranges even more narrow in case of narrow-index drugs. As a result, the following type of bioequivalence acceptance range is introduced:

$$
\begin{aligned}
U & =\alpha^{\prime \prime}+5\left(1-\frac{1}{\alpha^{\prime \prime}} \Psi\right)\left(\beta-\alpha^{\prime \prime}\right)\left\{1-e^{-\left(\gamma \sigma_{w}\right)^{2}}\right\}\left\{1-e^{-\left(\delta \frac{M T D}{D}\right)^{2}}\right\} 1_{\Psi \leq \alpha "}, \\
L & =\frac{1}{\alpha^{\prime}+5\left(1-\frac{1}{\alpha^{\prime}} \Psi\right)\left(\beta-\alpha^{\prime}\right)\left\{1-e^{-\left(\gamma \sigma_{w}\right)^{2}}\right\}\left\{1-e^{-\left(\delta \frac{D}{L E D}\right)^{2}}\right\} 1_{\Psi \leq \alpha^{\prime}},} \\
\alpha^{\prime} & =1+(\alpha-1)\left\{1-e^{-\left(\theta\left(1+\frac{D}{L E D}\right)\right)^{2}}\right\}, \\
\alpha^{\prime \prime} & =1+(\alpha-1)\left\{1-e^{-\left(\theta\left(1+\frac{M T D}{D}\right)\right)^{2}}\right\},
\end{aligned}
$$

where, as before, $\delta, \gamma$, and $\theta$ are rate constants. 


\section{Simulation Study}

The proposed bioequivalence acceptance ranges (2.5) depend on the therapeutic window as well as on the within-subject variability. These parameters, as well as the influence of the choice of the parameters $\delta, \theta$, and $\gamma$, are explored through simulations.

In the first simulation run, the acceptance ranges are calculated using (2.5) to explore their behavior for different values of $\theta, \delta$, the ratios $M T D / D$ and $D / L E D$, and the withinsubject variability with $\Psi=1$. The within-subject variability is presented as a coefficient of variation $(\% C V)$ in line with pharmacokinetics practice. It is linked to the within-subject variability, as follows:

$$
\sigma_{w}=\sqrt{\ln \left(1+\% C V^{2}\right)}
$$

Figure 1 shows the new acceptance range for different choices of $\delta$ and $\theta$ as a function of the ratio $M T D / D$ for the upper limit and $D / L E D$ for the lower limit. It shows that, for each choice of $\delta$ and $\theta$, the upper or the lower acceptance limit is reduced when the tested dose approaches the boundary of the therapeutic window, i.e., when $M T D / D$ or $D / L E D$ approach unity. For doses far from the boundary of the therapeutic window, the ratios $M T D / D$ and $D / L E D$ are larger and the acceptance ranges broaden (righthand side of the graphs). This is a conservative approach to ensure patients maintain a safe and efficacious exposure. With $\theta$ increasing from 0.1 to 1 , the slopes of the acceptance ranges become steeper near the therapeutic borders. A value of 0.3 for $\theta$ seems reasonable: the resulting shallow slope protects patients by imposing strict acceptance limits close to the borders of the therapeutic window. For a higher value of $\theta$, the influence of the ratios $M T D / D$ and $D / L E D$ vanishes and they may not be sufficiently conservative.

Whereas the parameter $\theta$ regulates the shrinkage of the acceptance range with respect to the therapeutic window, the parameter $\delta$ determines the expansion of the limits as a function of the therapeutic window and the within-subject variability. It basically means that for a highly variable drug product with a dose near the boundaries of the therapeutic window, the expansion of the acceptance limits is smaller than the ones proposed by Karalis 
et al. (2005). For a dose far from the therapeutic boundary, the acceptance ranges behave similar to the ones in the aforementioned article.

A small value for $\delta$, e.g. 0.1, penalizes the acceptance ranges in a very conservative way, whereas values ranging over $0.7-1$ are too liberal and impose little restriction (Figure 1). Therefore, an intermediate value of 0.4 for $\delta$ seems reasonable.

Although not demonstrated in the figures, the approach of Karalis et al. (2005) is maintained and extended: the acceptance ranges depend on the within-subject variability of the drug products and gradually expand from $80-125 \%$ to $70-143 \%$, as a function of the within-subject variability.

Whereas the previous calculations mainly illustrated the general concepts of the new approach to acceptance limits, the ensuing set of simulations was performed to compare it to existing methods (Figure 2). Thousand two-treatment, two-period cross-over studies with 36 subjects where simulated per condition, defined by the within-subject variability (\% $C V$ of $15 \%, 35 \%$, and $55 \%$ ), and the true geometric mean ratio $\Psi$ (100\% to $150 \%)$. $\theta$ was fixed at 0.3 , and $\delta$ set to 0.4 . The simulation was simplified in a first step by setting $M T D / D$ equal to $D / L E D$. These ratios varied from 1 to 10 in the simulation, to cover a broad spectrum of therapeutic windows. Simulations were performed using SAS 9.1 and analyzed with procedure MIXED.

Our acceptance limits and those obtained by the method of Karalis et al. coincided for $35 \% \mathrm{CV}$ for a $M T D / D$ ratio from 7 onwards. For a narrow-index drug, e.g., a $M T D / D$ of 3 or less, the acceptance rate is strongly decreased due to desired shrinkage of the acceptance limits. For low-variable drugs $(15 \% \mathrm{CV})$, the methods are essentially equivalent to the Schuirmann method, but for highly variable drug products $(55 \% \mathrm{CV})$, the methods give clear differences. The proposed method behaves as liberal as the Karalis' method for drugs with a broad therapeutic window, and more conservative than Schuirmann for narrow-index drugs. The simulations also indicate that only a very small amount of the studies with $\Psi$ superior to $125 \%$ conclude bioequivalence as would be expected based on (2.5). For any $\Psi$, the acceptance rate increases with the therapeutic window. Further exploration of the effect of 
the sample size and changes of the within-subject variability in Figures 3 and 4 confirmed the previous conclusions.

Table 1 contains a summary of the above simulations for the specific case of $\Psi=125 \%$, i.e. the point from where onwards bioequivalence is rejected in classic bioequivalence testing. It represents the proportion of simulated trials where bioequivalence is concluded, whereas in fact the two products are bio-inequivalent. This corresponds to the type- 1 error for the Schuirmann method. These values are larger than for Schuirmann, but correspond to the method of Karalis for $M T D / D$ large, but they decrease well below the Schuirmann error rate when the dose approaches the $M T D$. Therefore the new acceptance limits are conservative when it is in the patients interest. This illustrates well the strength of the method.

Figures $5-7$ represent the same simulations as before, but now for a situation where the dose is closer to the $L E D$ than the $M T D$, where $D / L E D$ varies from 1 to 10 and $M T D / D$ is fixed to 10 . When the dose is close to the $L E D$, i.e. less than 3 , the number of accepted trials when $\Psi=100 \%$ was lower than when $\Psi=105 \%$. Here, the conservative nature of the acceptance limits clearly distinguishes our method from Schuirmann's and Karalis' methods: The asymmetry of the limits render many trials inconclusive for a true $\Psi$ of $100 \%$, whereas this is not the case for $\Psi=105 \%$. This ensures patients will maintain an efficacious exposure.

\section{Application}

The Canadian health authorities recently published a guideline for critical dose drugs (Ministry of Health Canada, 2006). In the appendix of the guideline, a list can be found with a number of drug substances for which a small difference in dose or concentration lead to doseand concentration-dependent, therapeutic failure and/or serious adverse drug reactions: cyclosporine, digoxin, flecainide, lithium, phenytoin, sirolimus, tracolimus, theophylline, and warfarin. For these drug substances, the more stringent $90-112 \%$ acceptance limits for $A U C$ in case of single dose administration are proposed. The three examples below illustrate well 
the conservative behavior of the new acceptance limits for narrow index drugs.

\subsection{Theophylline}

Also theophylline belongs to the list of critical-dose drugs (Ministry of Health Canada, 2006). The data of Mistry et al (1999) is reanalyzed with the different techniques. Note that the study was not fully powered to demonstrate a drug interaction of indinavir on a single dose of $250 \mathrm{mg}$ theophylline immediate release.

Again, the MTD and LED of theophylline can be derived from the literature. Theophylline therapeutic plasma concentrations range from 10 to $20 \mu \mathrm{g} / \mathrm{mL}$, seizures and cardiac problems can occur at the upper limit (Ministry of Health Canada, 2006). Estimates of the first-order compartmental model $\left(k_{e}, k_{a}\right.$ rate constants, $C L$ clearance) were obtained from Pinheiro and Bates (2000): $\log \left(k_{e}\right)=-2.4327, \log \left(k_{a}\right)=-0.45146$, and $\log (C L)=-3.2145$, where dose was denoted in $\mathrm{mg} / \mathrm{kg}$. The accumulation factor for multiple dosing is $1 /\left(1-\exp \left(-k_{e} \tau\right)\right), \tau$ corresponding to 8 hours. Solving the equations for a $C_{\text {max }}$ set equal to the above range limits yields an $L E D$ of $220 \mathrm{mg}$ and a $M T D$ of $450 \mathrm{mg}$ for a subject of $70 \mathrm{~kg}$. Estimates for the variability are derived from Steinijans et al (1995): $\% C V$ for $A U C$ is $12 \%, 20 \%$ for $C_{\max }$.

The conclusion based on the traditional analysis was an absence of a drug interaction effect: $1.18(1.13,1.23)$ for $A U C$, and $0.99(0.92,1.07)$ for $C_{\max }$ fall both within the 80-125\% Schuirmann acceptance ranges. As the Karalis acceptance limits are always broader or equal to Schuirmann, this method also brings us to concluding that a drug interaction is absent. Our new acceptance limits, taking the variability as well as the therapeutic window into account, were $(0.94,1.12)$ for $A U C$, and $(0.88,1.15)$ for $C_{\max }$. Therefore, the confidence interval for $A U C$ falls entirely outside the acceptance range and a drug interaction would be concluded for $A U C$. 


\subsection{Digoxin}

As a last example, digoxin is another critical dose drug (Ministry of Health Canada, 2006). Martin et al (1997) evaluated the drug interaction of eprosartan on $0.6 \mathrm{mg}$ digoxin. We reevaluate these study results with the new method.

Serum digoxin levels ranging from 0.8 to $2.0 \mathrm{ng} / \mathrm{mL}$ are generally considered as therapeutic. Levels greater than $2.0 \mathrm{ng} / \mathrm{mL}$ are often associated with toxicity (Ministry of Health Canada, 2006). The IV compartmental model as well as parameter estimates of digoxin for healthy volunteers are found in Wagner (1975). The bioavailability for tablets is $80 \%$ (Bochner et al 1977). Estimates for the variability of digoxin are derived from Steinijans et al (1995): \%CV for $A U C$ is $8 \%, 19 \%$ for $C_{\max }$. Using these estimates, the $M T D$ and $L E D$ of digoxin can be derived: $0.4 \mathrm{mg}$ as $L E D$, and $1 \mathrm{mg}$ as $M T D$.

The geometric mean ratio in the original analysis was $1.01(0.81,1.26)$ for $A U C$, and $1.00(0.86,1.17)$ for $C_{\max }$. Our new acceptance limits were $(0.90,1.12)$ for $A U C$, and $(0.90$, 1.13) for $C_{\max }$. Therefore the trial was inconclusive for both parameters.

\subsection{Phenytoin}

In this section, we will reanalyse a bioequivalence study using one of these critical-dose drugs (phenytoin) comparing the method of Karalis to our newly proposed acceptance limit. In Meyer (2001), three different lots of $100 \mathrm{mg}$ phenytoin sodium capsules were compared. In this study, the observed $\% \mathrm{CV}$ was low, i.e., $14 \%$ and $11 \%$ for $C_{\max }$ and $A U C$ respectively. The conclusion based on the traditional analysis was that all 3 lots were bioequivalent.

To apply our method, the MTD and LED of phenytoin were deduced from the literature. Phenytoin exhibits Michaelis-Menten kinetics, which is described by the following equation (Gibaldi and Perrier 1982) for the steady state plasma concentrations $c_{s s}$ :

$$
c_{s s}=\frac{F D}{\tau C L_{s}}
$$


where

$$
C L_{s}=\frac{V_{m} V}{K_{m}+c_{s s}}
$$

and $\tau$ represents the dosing interval. $C L_{s}$ is the clearance parameter, $V_{m}$ is the theoretical maximum rate of the process, $K_{m}$ the Michaelis constant, $V$ is the volume of distribution, and $F$ is the bioavailability. Estimates of the Michaelis-Menten constants $V_{m}$ and $K_{m}$ for phenytoin are reported as $17.87 \mathrm{mg} / \mathrm{h}$ and $4.29 \mathrm{mg} / \mathrm{L}$, respectively (Santos Buelga 2002). In the same study, an average steady state concentration $c_{s s}$ of $12.5 \mathrm{mg} / \mathrm{L}$ was observed after multiple dosing of $155 \mathrm{mg}$. Given the fact that phenytoin is traditionally prescribed as b.i.d., i.e., $\tau$ is set to 12 hours, solving (4.1) and (4.2) for the unknown apparent volume of distribution, leads to an estimate of

$$
V / F=\frac{D}{\tau} \frac{K_{m}+c_{s s}}{V_{m} c_{s s}}=0.97
$$

Phenytoin is associated with severe neurological toxicity from $160 \mu \mathrm{mol} / \mathrm{L}$ onwards, whereas therapeutic plasma concentrations range from 40 to $80 \mu \mathrm{mol} / \mathrm{L}$ (Ministry of Health Canada, 2006). Therefore, a dose associated with $160 \mu \mathrm{mol} / \mathrm{L}$ steady state plasma concentrations will be considered the $M T D$. Based on the above estimations and equations, one can now calculate the $M T D$ associated with $c_{s s}=160 \mu \mathrm{mol} / \mathrm{L}$, or $c_{s s}=43.9 \mathrm{mg} / \mathrm{L}$, given the molecular weight of phenytoin sodium $(274.3 \mathrm{~g} / \mathrm{mol})$. Solving again (4.1) and (4.2) for $D$ gives an MTD of $190 \mathrm{mg}$ b.i.d., or a total daily dose of $380 \mathrm{mg}$. Analogue, the lower limit of the therapeutic window is associated with $c_{s s}=40 \mu \mathrm{mol} / \mathrm{L}$, or $c_{s s}=10.975 \mathrm{mg} / \mathrm{L}$. This leads to an $L E D$ of $150 \mathrm{mg}$ b.i.d. This means that the dose tested in the study was lower than the $L E D$. However, drug-monitoring is required for phenytoin to ensure patients remain on an optimal exposure. Therefore, the dose corresponding to $60 \mu \mathrm{mol} / \mathrm{L}$ in an average patient will be considered as the therapeutic dose, i.e. $165 \mathrm{mg}$.

Table 2 contains the geometric mean ratio, its $90 \%$ confidence interval, the equivalence limits using Karalis' equation, and our newly proposed acceptance ranges. The conclusions do not change for the Karalis method and it is inconclusive for all but three cases with novel method because the lower limit of the confidence interval falls below the acceptance limit. This example illustrates that the technique of Karalis only expands the acceptance limits, 
whereas in our approach, the acceptance limits reduce if the dose is close or outside the edge of the therapeutic window.

\section{Discussion and Conclusions}

Bioequivalence testing is an important topic in drug development. In this kind of trials, the pharmacokinetic parameters $A U C$ and $C_{\max }$ serve as surrogate markers for safety and efficacy in the sense that the equivalence of the pharmacokinetic parameters between test and reference implicitly implies that test and reference products have equivalent efficacy and safety. To claim bioequivalence of the parameters, an acceptance range of $80-125 \%$ is predefined, which implicitly leads to the conclusion that the observed differences have no efficacy or safety repercussions.

However, the assumption that changes within the $80-125 \%$ range have no clinical implications ought to be verified. For narrow-index drugs, even an exposure change of $10 \%$ might affect safety and/or efficacy, whereas doubling the exposure for certain other drug products would not affect the safety at all. It is interesting to see that this idea was already reflected in the conclusion of Sheiner (1992):

"... The main point is that the logical basis for current bioequivalence measurement and regulation is seriously inadequate: only with an appropriate model for dose effect, and a clear delineation of clinical context and values, can one devise, estimate and test bioequivalence measures that make clinical and scientific sense. We should judge future contributions to the bioequivalence literature by how well they meet this requirement."

Since then, to our knowledge, no paper has addressed bioequivalence testing in this respect.

One might question the regulatory imposed acceptance ranges, since this approach treats all drug products in the same way. One of the concerns is that highly variable drug 
products, i.e., a within-subject variability of more than $30 \%$, are treated the same way as the rest. This results in studies with unpractical large sample sizes. Boddy (1995) and Karalis (2004, 2005) proposed, respectively, scaled average bioequivalence and bioequivalence with levelling-off properties. Both of these correct the acceptance ranges with respect to the within-subject variability, but do not answer the clinical relevance of the acceptance limits, and rather limits to the logistics and ethics of the method.

The newly proposed acceptance ranges take besides the within-subject variability also the therapeutic window into account. More specifically, the proposed approach is highly conservative for doses near the boundaries of the therapeutic window, defined by the ratios $M T D / D$ and $D / L E D$, and more liberal for doses far from the maximum tolerated dose and least effective dose.

A simulation study shows that for doses near the $M T D$, lower acceptance limits are imposed for the upper limit of the $90 \%$ confidence interval: this should ensure that patients will not experience toxic exposures for compounds with a narrow therapeutic window. The same recommended for doses close to the least effictive dose: the lower acceptance limit will approach $100 \%$ to ensure patients remain on active doses. On the other hand, for doses far from the boundaries of the therapeutic window, the acceptance limits approach the ones of Karalis et al. (2005).

Based on the simulations, it has been demonstrated that the newly proposed bioequivalence limits differentiate between narrow index drugs and drug products with a wide therapeutic window. They are very strict when it is of interest for the patient, and more flexible when the therapeutic effect remains unaffected. Traditional methods, on the contrary, apply a uniform method, regardless as to where the marketed dose is positioned in the therapeutic window.

Since the newly proposed bioequivalence limits depend on the $M T D$ and the $L E D$, these quantities need to be determined as accurately as possible in an early stage of drug development. This emphasizes the need for adequate dose finding trials using stochastic methods such as most prominently, the continuous reassessment method (O'Quigley et al. 
1990, Patterson et al. 1999). However, also literature can be a good source for estimates of the $M T D$ and the $L E D$ as illustrated in the application.

Financial support from the IAP Research Network P6/03 of the Belgian Government (Belgian Science Policy) is gratefully acknowledged.

\section{References}

Anderson, S., Hauck, W.; "Consideration of individual bioequivalence; Journal of Pharmacokinetics and Biopharmaceutics; 1990, Vol.18 (3), 259-273.

Bochner F., Huffman D., Shen D., Azarnoff D.; "Bioavailability of digoxin-hydroquinone complex: A new oral digoxin formulation"; Journal of Pharmaceutical Sciences; 1977, Vol.66 (5), 644-647.

Boddy, A., Snikeris, F., Kringle, R., Wei, G., Opperman, J., Midha, K.; "An approach for widening the bioequivalence limits in the case of highly variable drugs. Pharm. Res., 1995, vol 12, 1865-1868.

Committee for Proprietary Medicinal Products (CPMP), the European Agency for the Evaluation of Medicinal Products (EMEA); "Note for guidance on the investigation of bioavailability and bioequivalence "; 2001.

Committee for Proprietary Medicinal Products (CPMP), the European Agency for the Evaluation of Medicinal Products (EMEA); "Concept paper for an addendum to the note for guidance on the investigation of bioavailability and bioequivalence: evaluation of bioequivalence of highly variable drugs and drug products"; 2006.

U.S. Food and Drug Administration, Center for Drug Evaluation and Research; "Guidance for Industry: Bioavailability and Bioequivalence Studies for Orally Administered Drug Products — General Considerations"; 2003.

Gibaldi, M., Perrier, D.; "Pharmacokinetics"; New York, Marcel Dekker; 1982. 
Hsuan, F.; "Some statistical considerations on the FDA draft guidance for individual bioequivalence"; Statistics in Medicine; 2000; Vol 19, 2879-2884.

Karalis, V., Symillides, M., Macheras, P.; "Novel Scaled Average Bioequivalence Limits Based on GMR and Variability Considerations; Pharmaceutical Research; 2004, Vol 21 (10), 1933-1942.

Karalis, V., Macheras, P., Symillides, M.; "Geometric mean ratio-dependent scaled bioequivalence limits with leveling-off properties; Eur. J. Pharm. Sci.; 2005, Vol 26 (1), $54-61$.

Meyer M.C., Straughn A.B., Mhatre R.M., et al.; "Variability in the bioavailability of phenytoin capsules in males and females."; Pharm. Res.; 2001, Vol 18, 394-397.

Ministry of Health, Canada; "Bioequivalence requirements: Critical dose drugs"; 2006.

Mistry, G., Laurent, A., Sterrett, A., Deutsch, P.; "Effect of indinavir on the single-dose pharmacokinetics of theophylline in healthy subjects."; J. Clin. Pharmacol.; 1999, Vol 39, 636-642.

Martin, D., Tompson, D., Boike, S., Tenero, D., Ilson, B., Citerone, D., Jorkasky, D.; "Lack of effect of eprosartan on the single dose pharmacokinetics of orally administered digoxin in healthy male volunteers"; Br. J. Clin. Pharmacol.; 1997, vol 43, 661-664.

Pinheiro, J., Bates, D.; "Mixed-Effects Models in S and S-PLUS", Springer, 2000.

O'Quigley, J., Pepe, M., Fisher, L.; "Continual reassessment method: a practical design for phase I clinical trials in cancer; Biometrics; 1990, Vol 46, 33-48.

Patterson, S., Francis, S., Ireson, M., Webber, D., Whitehead, J.; "A novel Bayesian decision procedure for early-phase dose finding studies; J. Biopharmaceutical Statistics; 1999, Vol 9, 583-597.

Santos Buelga, D., Garcia, M.J., Otero, M.J., Martin Suarez, A., Dominguez-Gil, A., Lukas, J.C.; Phenytoin covariate models for Michaelis-Menten pharmacokinetics in adult epileptic patients; Page meeting, Paris, 2002; 
Sheiner, L.B.; "Bioequivalence revisited"; Stat. Med.; 1992, vol 11, 1777-1788.

Schuirmann, D.; "A comparison of the two one-sided tests procedure and the power approach for assessing the average bioavailability"; Journal of Pharmacokinetics and Biopharmaceutics 1987; 15:657-680.

Steinijans, V., Sauter, R., Hauschke, D., Diletti, E., Schall, R., Luus, H., Elze, M., Blume, H., Hoffmann, C., Franke, G., Siegmund, W.; "Reference tables for the intrasubject coefficient of variation in bioequivalence studies"; Int. j. clin. pharmacol. ther.; 1995, vol. $33,427-430$.

Wagner, J.; "Fundamentals of Clinical Pharmacokinetics", Drug Intelligence, 1975. 
Table 1: The proportion of simulated trials for which bioequivalence was concluded erroneously at $\Psi=125 \%$, as a function of $\% \mathrm{CV}$ and sample size, in the case of $M T D / D=$ $D / L E D=\mathcal{R}, \theta=0.3, \delta=0.4$, and $\gamma=3$.

\begin{tabular}{|c|c|c|c|c|c|c|c|c|c|}
\hline \multirow[b]{3}{*}{$\% \mathrm{CV}$} & \multirow{2}{*}{\multicolumn{3}{|c|}{$\begin{array}{c}\text { Sample size } \\
12\end{array}$}} & \multirow{2}{*}{\multicolumn{3}{|c|}{$\begin{array}{c}\text { Sample size } \\
24\end{array}$}} & \multirow{2}{*}{\multicolumn{3}{|c|}{$\begin{array}{c}\text { Sample size } \\
36\end{array}$}} \\
\hline & & & & & & & & & \\
\hline & 15 & 35 & 55 & 15 & 35 & 55 & 15 & 35 & 55 \\
\hline Schuirman & 0.0512 & 0.0190 & 0.0023 & 0.0475 & 0.0485 & 0.0066 & 0.0493 & 0.0529 & 0.0324 \\
\hline Karalis & 0.0515 & 0.0227 & 0.0028 & 0.0478 & 0.0636 & 0.0601 & 0.0497 & 0.0641 & 0.0976 \\
\hline $\mathcal{R}=10$ & 0.0515 & 0.0227 & 0.0028 & 0.0478 & 0.0636 & 0.0601 & 0.0497 & 0.0641 & 0.0976 \\
\hline $\mathcal{R}=7$ & 0.0503 & 0.0225 & 0.0028 & 0.0468 & 0.0624 & 0.0591 & 0.0474 & 0.0637 & 0.0970 \\
\hline $\mathcal{R}=5$ & 0.0394 & 0.0177 & 0.0021 & 0.0327 & 0.0539 & 0.0458 & 0.0308 & 0.0545 & 0.0876 \\
\hline $\mathcal{R}=3$ & 0.0083 & 0.0037 & 0.0001 & 0.0027 & 0.0171 & 0.0020 & 0.0013 & 0.0184 & 0.0324 \\
\hline $\mathcal{R}=2$ & 0.0007 & 0.0002 & 0 & 0 & 0.0005 & 0 & 0 & 0.0022 & 0 \\
\hline $\mathcal{R}=1$ & 0 & 0 & 0 & 0 & 0 & 0 & 0 & 0 & 0 \\
\hline
\end{tabular}

Table 2: Reconsidering the bioequivalence testing of Phenytoin using the data from Meyer (2001) .

\begin{tabular}{llccrc}
\hline \hline & $\begin{array}{l}\text { test vs } \\
\text { reference }\end{array}$ & $\Psi$ & $\begin{array}{c}90 \% \text { confidence } \\
\text { interval }\end{array}$ & $\begin{array}{r}\text { Karalis } \\
\text { limit }\end{array}$ & $\begin{array}{c}\text { New } \\
\text { limit }\end{array}$ \\
\hline \hline$C_{\max }$ & 2 vs 1 & 0.986 & $(0.90 ; 1.04)$ & $(0.781 ; 1.280)$ & $(0.921 ; 1.090)$ \\
& 3 vs 1 & 0.993 & $(0.92 ; 1.05)$ & $(0.781 ; 1.280)$ & $(0.921 ; 1.090)$ \\
& 4 vs 1 & 0.979 & $(0.89 ; 1.02)$ & $(0.780 ; 1.281)$ & $(0.920 ; 1.090)$ \\
& 3 vs 2 & 0.995 & $(0.92 ; 1.06)$ & $(0.782 ; 1.279)$ & $(0.921 ; 1.090)$ \\
& 4 vs 2 & 0.993 & $(0.92 ; 1.05)$ & $(0.781 ; 1.280)$ & $(0.921 ; 1.090)$ \\
& 4 vs 3 & 0.988 & $(0.91 ; 1.04)$ & $(0.781 ; 1.280)$ & $(0.921 ; 1.090)$ \\
AUC 2 vs 1 & 0.975 & $(0.90 ; 0.99)$ & $(0.787 ; 1.270)$ & $(0.922 ; 1.089)$ \\
& 3 vs 1 & 0.997 & $(0.95 ; 1.04)$ & $(0.788 ; 1.269)$ & $(0.922 ; 1.088)$ \\
& 4 vs 1 & 0.984 & $(0.92 ; 1.01)$ & $(0.788 ; 1.270)$ & $(0.922 ; 1.088)$ \\
3 vs 2 & 0.980 & $(0.91 ; 1.00)$ & $(0.787 ; 1.270)$ & $(0.922 ; 1.088)$ \\
& 4 vs 2 & 0.991 & $(0.93 ; 1.03)$ & $(0.788 ; 1.269)$ & $(0.922 ; 1.088)$ \\
4 vs 3 & 0.989 & $(0.93 ; 1.02)$ & $(0.788 ; 1.269)$ & $(0.922 ; 1.088)$ \\
\hline \hline
\end{tabular}


delta $=0.1 \quad \% C V=30$

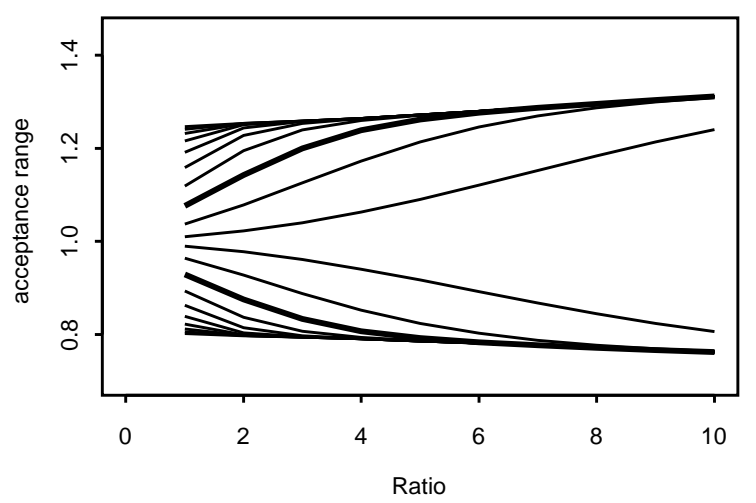

delta $=0.7 \quad \% \mathrm{CV}=30$

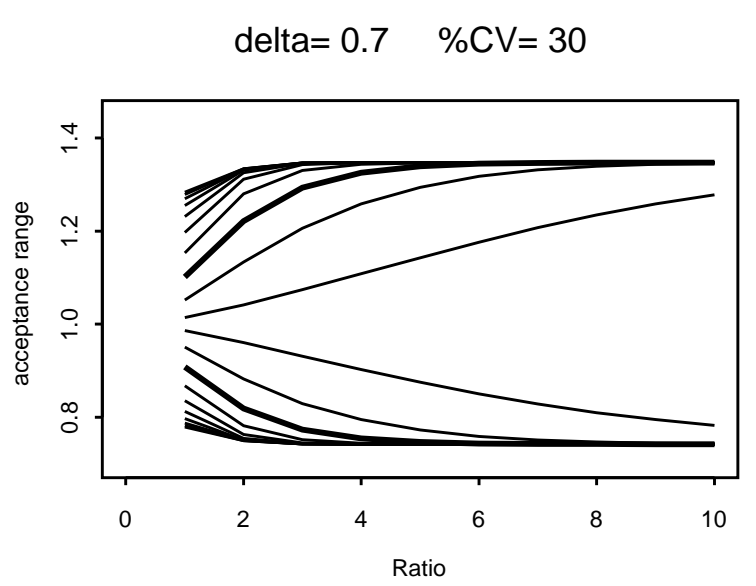

delta $=0.4 \quad \% \mathrm{CV}=30$

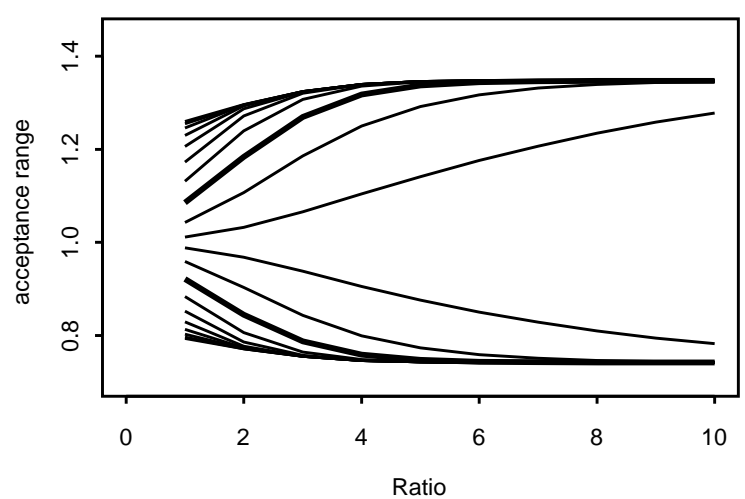

Figure 1: Illustration of the influence of therapeutic window by varying $\theta$ from 0.1 (middle) to 1 (outside) on the newly proposed bioequivalence acceptance range for different $\delta$. The tick line represents the case $\theta=0.3$. For the upper limit, the ratio in the $\mathrm{x}$-axis represents $M T D / D$ whereas $D / L E D$ for the lower limit. A $\% \mathrm{CV}$ of $30 \%$ was assumed. 
$C V=15 \%$

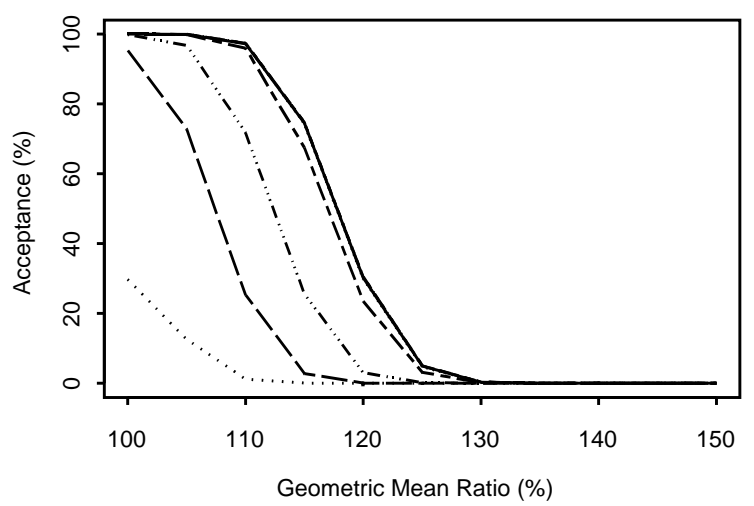

$\mathrm{CV}=55 \%$

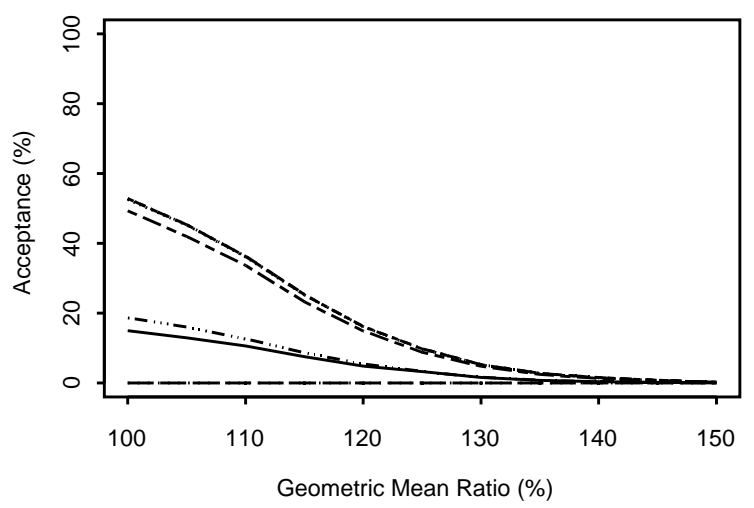

$C V=35 \%$

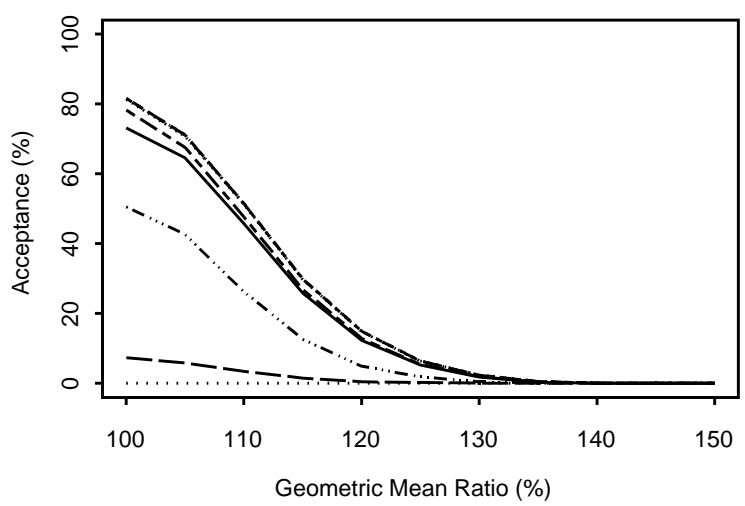

- Schuirmann Karalis Ratio = 1

- Ratio $=2$

- ...- Ratio $=3$

- - $\quad$ Ratio $=5$

$\begin{array}{ll}\ldots . . . . . & \text { Ratio }=7 \\ \ldots . .- & \text { Ratio }=10\end{array}$

Figure 2: Influence of the within-subject variability on the acceptance (\%) of bioequivalence trials using Schuirmann's method, Karalis and our new proposal with $M T D / D=D / L E D$ from 1 to 10 . The sample size is fixed to 36 subjects. 
$C V=15 \%$

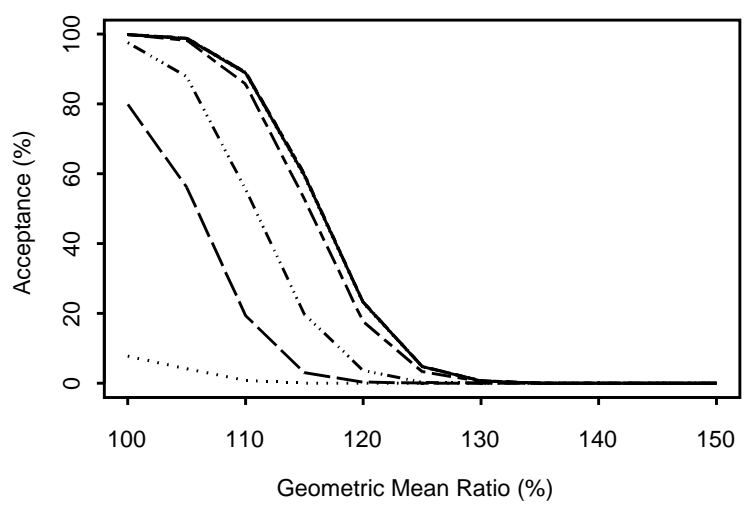

$\mathrm{CV}=55 \%$

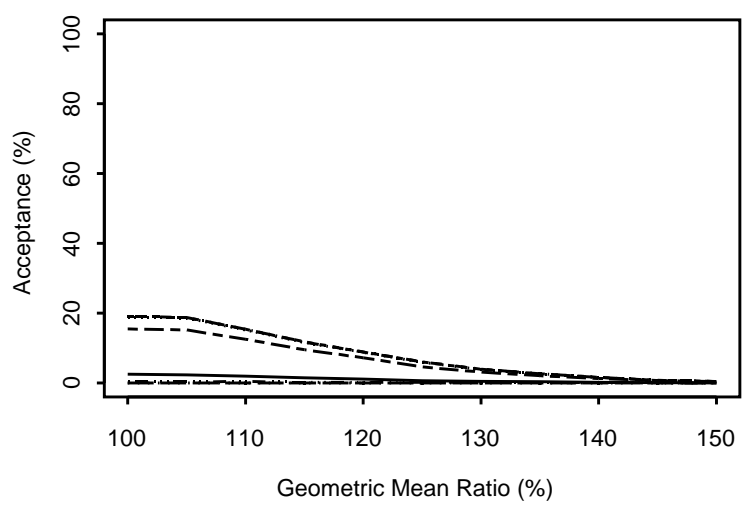

$C V=35 \%$

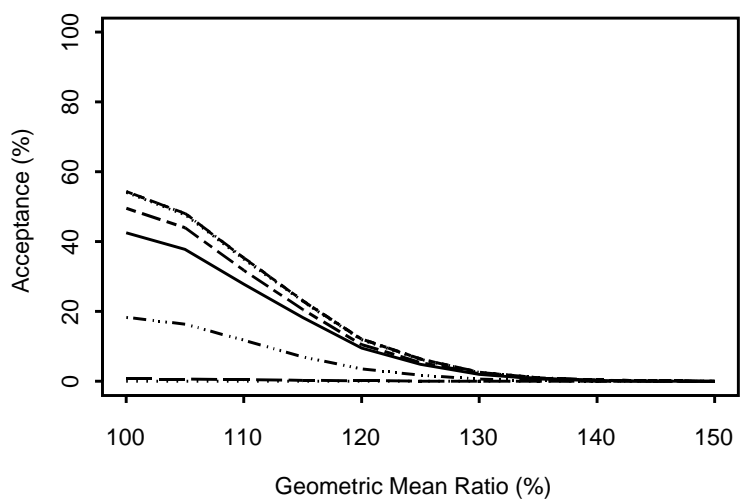

- Schuirmann Karalis Ratio = 1

- Ratio $=2$

- -...- Ratio $=3$

- - Ratio $=5$

$\begin{array}{ll}\ldots . . . . . & \text { Ratio }=7 \\ \ldots . .- & \text { Ratio }=10\end{array}$

Figure 3: Influence of the within-subject variability on the acceptance (\%) of bioequivalence trials using Schuirmann's method, Karalis and our new proposal with $M T D / D=D / L E D$ from 1 to 10 . The sample size is fixed to 24 subjects. 

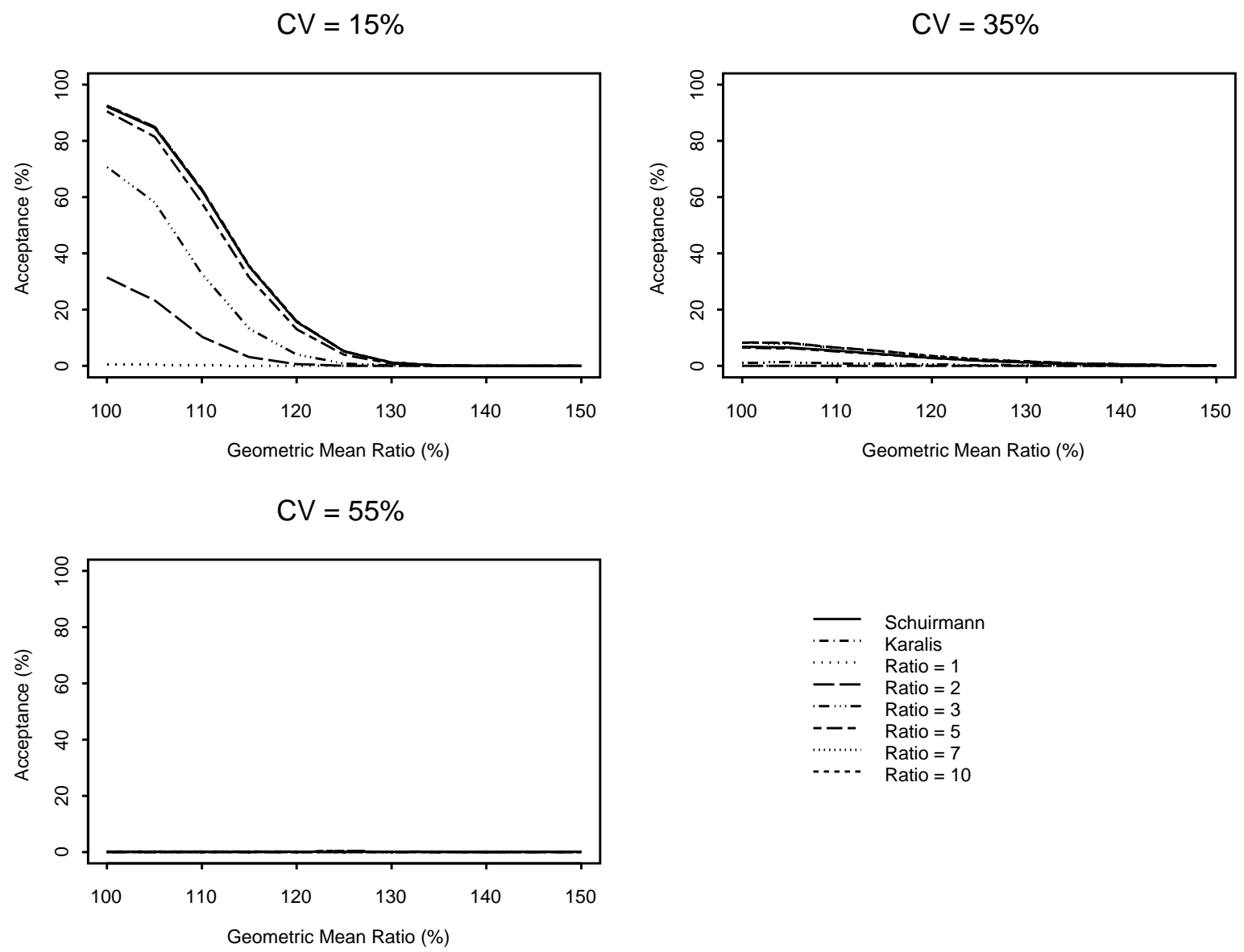

Figure 4: Influence of the within-subject variability on the acceptance (\%) of bioequivalence trials using Schuirmann's method, Karalis and our new proposal with $M T D / D=D / L E D$ from 1 to 10 . The sample size is fixed to 12 subjects.. 
$\% \mathrm{CV}=15 \%$

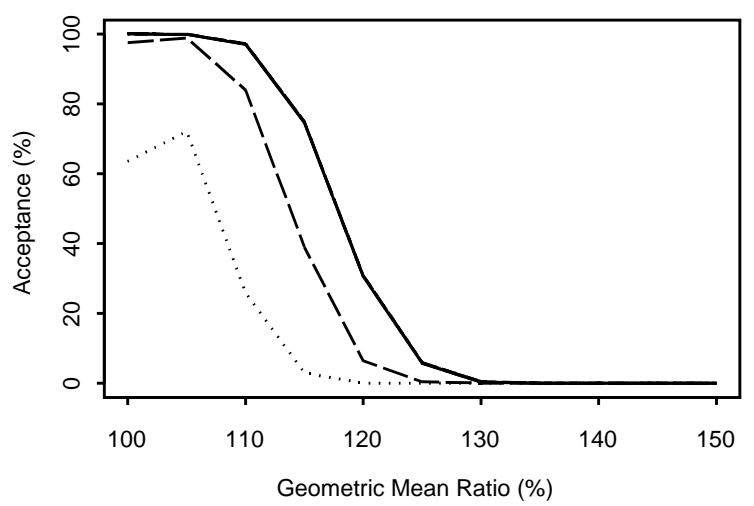

$\% \mathrm{CV}=55 \%$

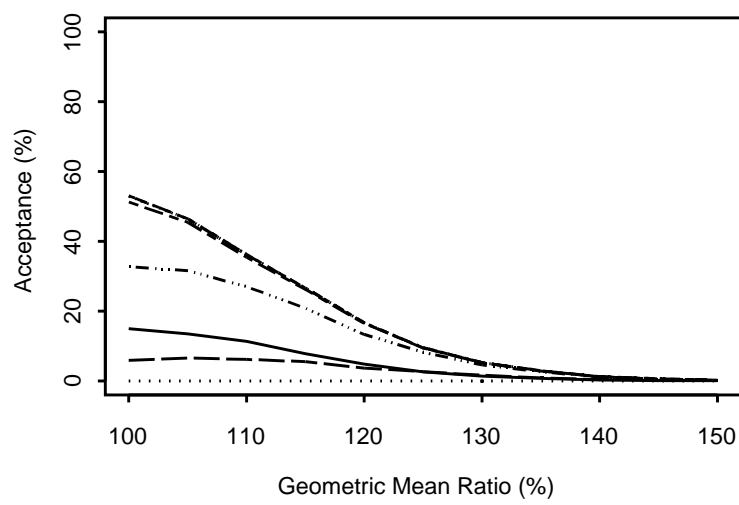

$\% \mathrm{CV}=35 \%$

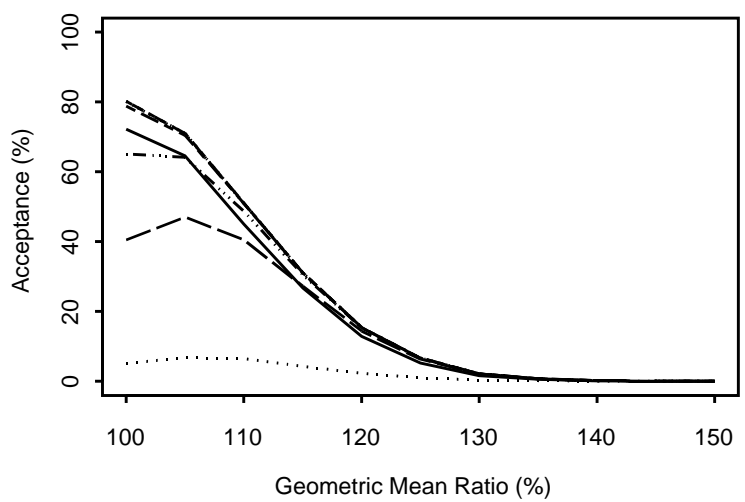

- Schuirmann Karalis Ratio = 1

- Ratio $=2$

- ...- Ratio $=3$

- - Ratio $=5$

$\begin{array}{ll}\ldots . . . . . & \text { Ratio }=7 \\ \ldots . .- & \text { Ratio }=10\end{array}$

Figure 5: Influence of the within-subject variability on the acceptance (\%) of bioequivalence trials using Schuirmann's method, Karalis and our new proposal with only $D / L E D$ from 1 to 10 and $M T D$ considered large. The sample size is fixed to 36 subjects. 
$\% \mathrm{CV}=15 \%$

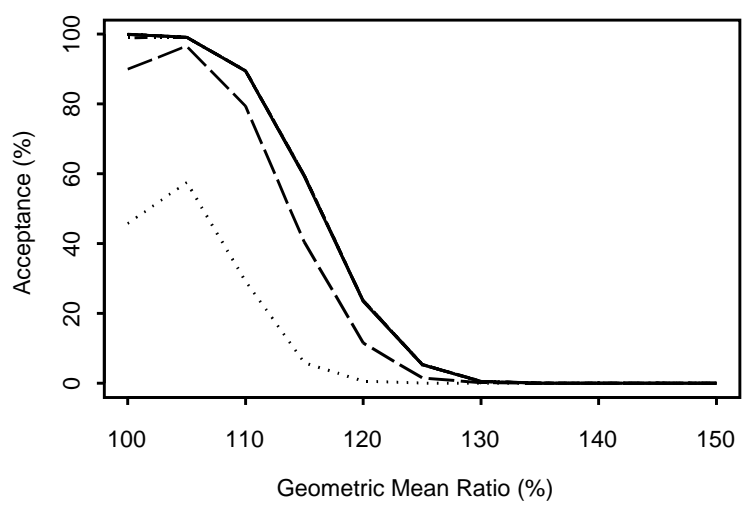

$\% \mathrm{CV}=55 \%$

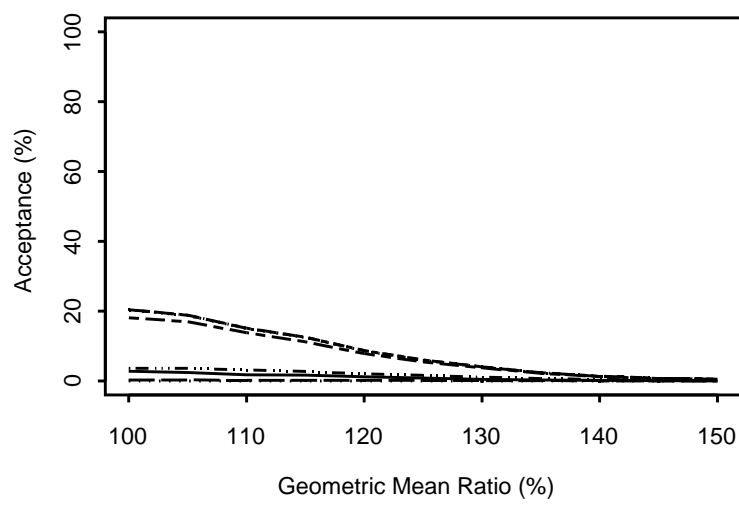

$\% \mathrm{CV}=35 \%$

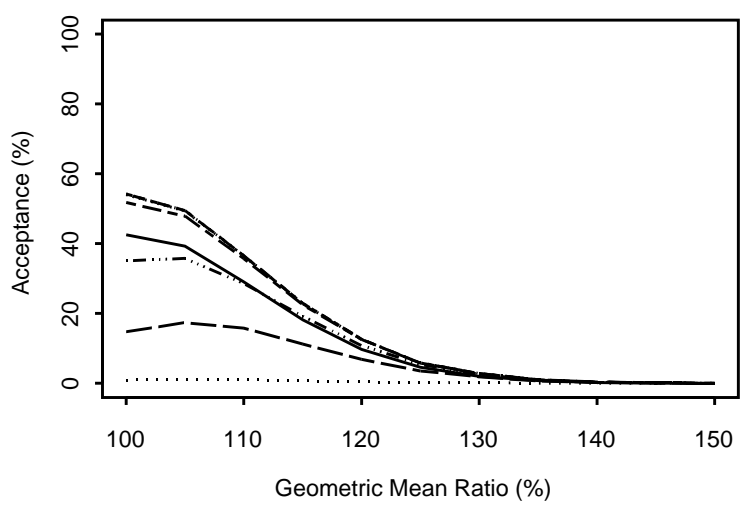

_... Schuirmann Karalis Ratio = 1

- Ratio $=2$

- $\cdots-$ Ratio $=3$

- - Ratio $=5$

$\begin{array}{ll}\ldots . . . . . & \text { Ratio }=7 \\ \ldots . .- & \text { Ratio }=10\end{array}$

Figure 6: Influence of the within-subject variability on the acceptance (\%) of bioequivalence trials using Schuirmann's method, Karalis and our new proposal with only $D / L E D$ from 1 to 10 and $M T D$ considered large. The sample size is fixed to 24 subjects. 

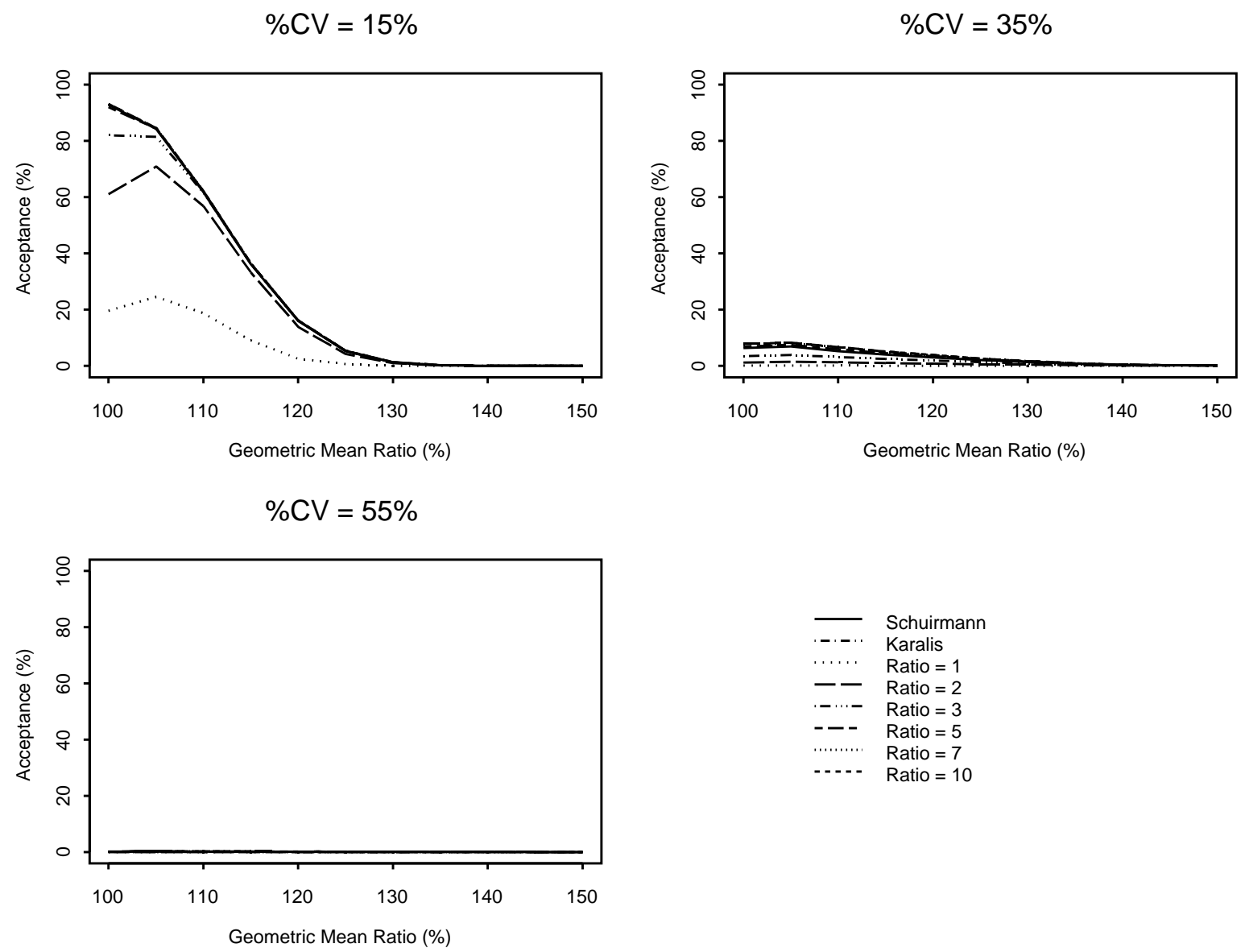

Figure 7: Influence of the within-subject variability on the acceptance (\%) of bioequivalence trials using Schuirmann's method, Karalis and our new proposal with only $D / L E D$ from 1 to 10 and $M T D$ considered large. The sample size is fixed to 12 subjects. 